\title{
Factors that Affect Version of the J-Taper Stem
}

\author{
Kawakami H ${ }^{1 \#}$, Setoguchi $\mathrm{T}^{2 \#^{*}}$, Ishidou $\mathrm{Y}^{3}$, Nagano $\mathrm{S}^{1}$, Kakoi $\mathrm{H}^{1}$, Izumi ${ }^{1}$, Nakamura $\mathrm{S}^{1}$, Tominaga $\mathrm{H}^{1}$ \\ and Komiya $\mathrm{S}^{2}$
}

${ }^{1}$ Department of Orthopaedic Surgery, Graduate School of Medical and Dental Sciences, Kagoshima University, Kagoshima, Japan

${ }^{2}$ The Near-Future Locomotor Organ Medicine Creation Course (Kusunoki Kai), Graduate School of Medical and Dental Sciences, Kagoshima University, Kagoshima, Japan

${ }^{3}$ Department of Medical Joint Materials, Graduate School of Medical and Dental Sciences, Kagoshima University, Kagoshima, Japan

${ }^{*}$ Corresponding author: Takao Setoguchi, The Near-Future Locomotor Organ Medicine Creation Course (Kusunoki Kai), Graduate School of Medical and Dental Sciences, Kagoshima University, 8-35-1 Sakuragaoka, Kagoshima 890-8520, Japan, Tel: +81992755381; Fax: +81992654699; E-mail: setoro@m2.kufm.kagoshima-u.ac.jp

\#These two authors contributed equally to this study.

Received date: February 15, 2017; Accepted date: February 24, 2017; Published date: February 27, 2017

Citation: Setoguchi T, Yahiro Y, Ishidou Y, Nagano S, Kakoi H, et al. (2017) Factors that Affect Version of the J-Taper Stem. J Clin Exp Orthop 3: 32. doi: 10.4172/2471-8416.100032

Copyright: (C) 2017 Kawakami $\mathrm{H}$, et al. This is an open-access article distributed under the terms of the Creative Commons Attribution License, which permits unrestricted use, distribution, and reproduction in any medium, provided the original author and source are credited.

\section{Abstract}

Background: The appropriate combination of acetabular component and stem anteversion is needed to reduce implant impingement and dislocation of total hip arthroplasty (THA). J-Taper is a tapered wedge stem with a relatively shorter small area of arc spray coating compared with conventionally tapered wedge stems. We examined the factors that affect postoperative version of J-Taper.

Methods: A total of 59 patients who underwent primary THA were assessed by computed tomography. Preoperative femoral neck and postoperative stem anteversion, stem sagittal alignment, and stem coronal alignment were measured.

Results: Mean femoral neck anteversion was $26.1^{\circ} \pm 12.2^{\circ}$. Mean stem version was $32.3^{\circ} \pm 9.0^{\circ}$. Multiple linear regression analysis showed that femoral neck anteversion $(p<0.01)$, stem sagittal alignment $(p=0.045)$, and body height $(p=0.048)$ were positively correlated with stem aversion. The stem version was significantly increased compared with that of native femoral anteversion $\left(6.3^{\circ} \pm\right.$ $\left.10.9^{\circ}\right)$. Multiple linear regression analysis showed that femoral neck anteversion was negatively correlated with an increased angle of stem version, body height, and stem sagittal alignment was positively correlated.

Conclusions: Our findings are helpful for surgeons during preoperative planning to achieve the appropriate combined anteversion.

Keywords: J-Taper; Total hip arthroplasty (THA); Stem version; Combined anteversion

\section{Introduction}

Total hip arthroplasty (THA) is required to reconstruct the hip joint with a pain-free maximum range of motion without dislocation. Malalignment of the prosthesis promotes accelerated wear, dislocation, and revision [1,2]. Combined acetabular component and stem anteversion has been reported to reduce implant impingement and dislocation $[3,4]$. Short cementless stems have been developed to preserve the proximal bone stock and optimize proximal load transfer. Although this concept is not novel, there are several benefits, including easier insertion through smaller incisions and less invasive techniques, simpler femoral preparation with "broaching only," and more favorable conditions in the potential revision setting [5-7]. Several studies have demonstrated overall survivorship of short stems at $94-100 \%$ after up to 18 years of follow-up [7-9]. Although short stems have achieved adequate results, the mean prevalence of stem malalignment was $0-9.9 \%$ $[10,11]$. J-Taper was developed as a relatively shorter tapered wedge stem with a smaller area of arc spray coating than conventional tapered wedge stems (Figure 1). We examined postoperative J-Taper version using computed tomography (CT). We identified factors that affect postoperative version of J-Taper.

\section{Methods}

\section{Demographic data of patient}

We performed a retrospective study using preoperative and postoperative CT scans. The study group comprised 59 hips who had undergone primary THA by three surgeons (TS, YI, SK) at our institution between February 2013 and October 2014 using a short tapered stem (J-Taper; Kyocera Medical, Osaka, Japan). Patients with a history of femoral osteotomy were excluded because of changes in the femoral anatomy. The remaining 59 hips in 52 patients (15 men, 37 women) were included. The 
mean age at operation was 54.8 years (range $21-77$ years), and the mean body mass index was $23.5 \mathrm{~kg} / \mathrm{m} 2$ (range 16.3-32.7 $\mathrm{kg} / \mathrm{m}^{2}$ ). The preoperative diagnosis was osteoarthritis in 30 hips, osteonecrosis of the femoral head in 27 hips, rapidly destructive coxopathy in 1 hip, and rheumatoid arthritis in 1 hip.

\section{Measurements}

A ceramic-on-polyethylene bearing was used for all hips. Preoperative femoral neck anteversion and postoperative evaluation of the J-Taper were performed on CT images using three-dimensional template software according to the manufacturer's protocol (Kyocera Medical) [12]. Briefly, a threedimensional model was constructed with CT scans (HiSpeed Dx/i; GE Medical Systems, Milwaukee, WI, USA). The femoral axis was projected onto the retrocondylar plane. The retrocondylar plane of the femur included the most posterior points of the medial and lateral femoral condyles [13-16] and the most posterior point of the greater trochanter. The femoral axis was the line between the knee center and the trochanteric fossa. The $X$ axis was defined as perpendicular to the retrocondylar plane. The $Y$ axis was defined as the line on which the femoral axis was projected onto the retrocondylar plane. The $Z$ axis was defined as perpendicular to the $X$ and $Y$ axes [12]. The proximal femoral bone axis was defined as the line between the center of the femoral canal at the level of the lesser trochanter and the center of the femoral canal at the level of the isthmus. The femoral stem axis was defined as the axis of the distal half of the stem (Figure 1).
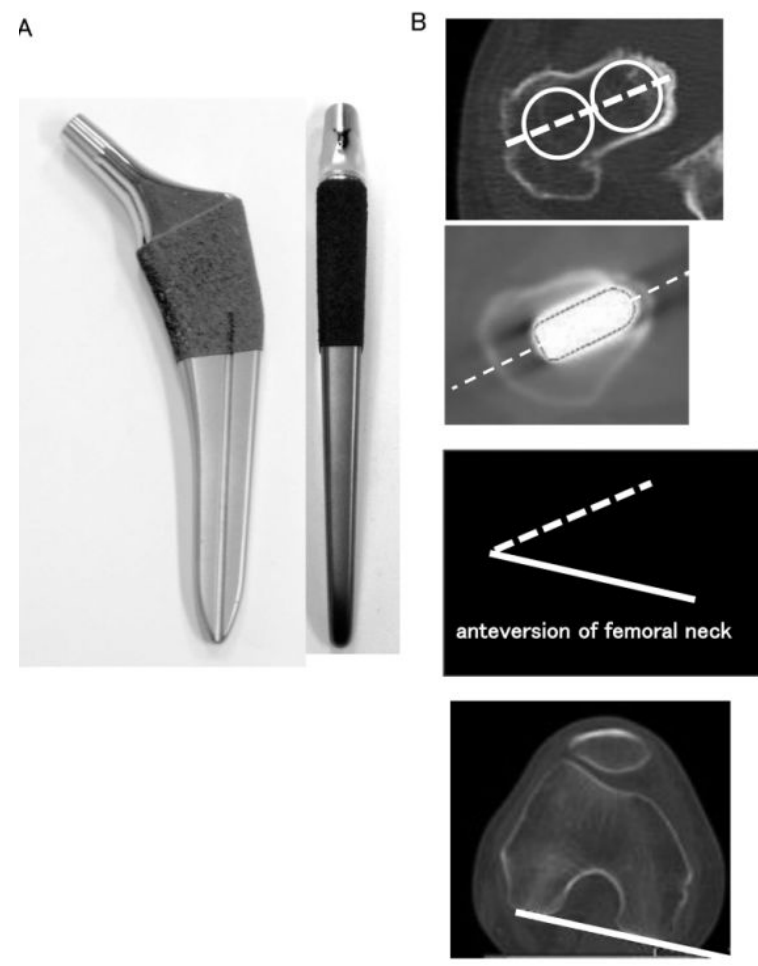

Figure 1: Evaluation of anteversion of femoral neck.

(A) Shape of the J-Taper stem. (B) A line between the centers of the circles that represented the femoral cortex and femoral head was drawn. The angle between these lines was defined as preoperative and postoperative stem anteversion. Anteversion of the preoperative femoral neck and postoperative stem was measured on the axial plane $1 \mathrm{~cm}$ above the level from the superior end of the lesser trochanter. A line between the centers of the circles that represented the femoral cortex and femoral head was drawn. The angle between the line and the $Z$ axis was defined as preoperative and postoperative stem anteversion.

The change in anteversion was measured as postoperative anteversion $\left({ }^{\circ}\right)$-preoperative anteversion $\left({ }^{\circ}\right)$. The stem's sagittal alignment was measured as the angle between the femoral tilt and the stem sagittal tilt on the sagittal plane: stem sagittal alignment $\left({ }^{\circ}\right)=$ stem sagittal alignment $\left({ }^{\circ}\right)$-femoral tilt $\left({ }^{\circ}\right)$. The positive and negative stem's sagittal axis values were defined (Figure 2). The coronal alignment of the femoral stem was defined as the difference between femoral lateral bowing and the stem coronal angle on the coronal plane: stem coronal alignment $\left({ }^{\circ}\right)=$ stem coronal angle $\left({ }^{\circ}\right)$-femoral lateral bowing $\left({ }^{\circ}\right)$ as previously reported. The positive and negative coronal stem axis values were defined (Figure 2 ).
A
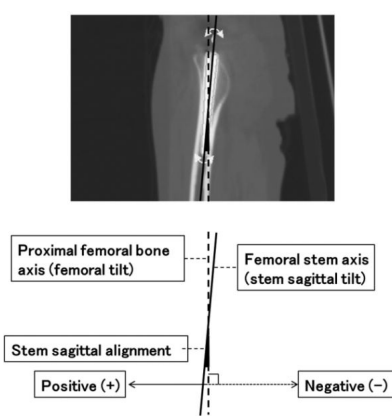

B

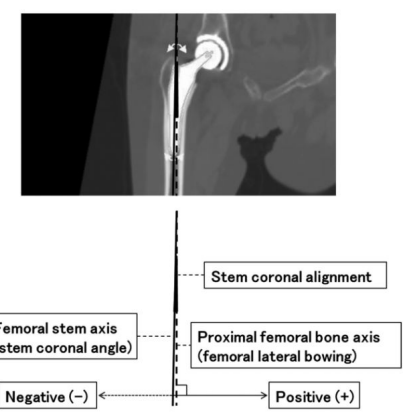

Figure 2: Evaluation of stem sagittal and coronal alignment. (A) Stem sagittal alignment on the sagittal plane (Stem sagittal alignment=stem sagittal tilt-femoral tilt). (B) Stem coronal alignment on the coronal plane (Stem coronal alignment=stem coronal angle-femoral lateral bowing).

We examined the intra- and inter-observer reliability of two of the authors' (HK, TS) evaluations of 25 consecutive hips. Intraobserver reliability was excellent (0.979-0.878), as was the interobserver reliability $(0.925-0.865)$.

\section{Statistical analysis}

A paired t-test was used to evaluate the difference between native anteversion of femoral neck and stem version. Spearman rank correlation coefficient was used to identify factors that related to stem anteversion or increased stem anteversion compared with femoral neck anteversion.

We evaluated eight factors, including native femoral neck version, length of the femur, stem anteversion, increased stem version, stem sagittal alignment, stem coronal alignment, body height, and body weight. Following Spearman's correlation coefficient test, we selected five factors-anteversion of the femoral neck, stem version, stem lateral alignment, stem coronal 
alignment, body height-as explanatory variables for step-wise multiple linear regression analysis of stem anteversion and increased anteversion of stem. Analysis was performed using BellCurve for Excel (Social Survey Research Information Co., Ltd. Tokyo, Japan). Values of $p<0.05$ were considered to indicate statistical significance.

\section{Ethics Statement}

The Ethics Committee on Clinical Research at our University approved this research protocol.

\section{Results}

The demographic data are summarized (Table 1). The measured values are summarized.

Table 1: Patients' characteristics.

\begin{tabular}{|l|l|l|}
\hline Characteristic & Number & Percentage \\
\hline Male & 18 & $30.5 \%$ \\
\hline Female & 41 & $69.5 \%$ \\
\hline Osteoarthritis & 30 & $50.8 \%$ \\
\hline Osteonecrosis of the femoral head & 27 & $45.8 \%$ \\
\hline Rapidly destructive coxopathy & 1 & $1.7 \%$ \\
\hline Rheumatoid arthritis & 1 & $1.7 \%$ \\
\hline & average & range \\
\hline Age & $54.8 \pm 15.4$ & $21-77$ \\
\hline Body height (cm) & $157.4 \pm 9.2$ & $135.0-175.6$ \\
\hline Body weight (Kg) & $57.9 \pm 10.1$ & $37.0-82.2$ \\
\hline Body mass index (kg/m2) & $23.5 \pm 3.6$ & $16.3-32.7$ \\
\hline Femoral length (mm) & $350.7 \pm 25.8$ & $290.6-412.2$ \\
\hline
\end{tabular}

The mean anteversion of the femoral neck was $26.1^{\circ} \pm 12.2^{\circ}$. The mean stem version was $32.3^{\circ} \pm 9.0^{\circ}$. Spearman's correlation coefficient test showed that anteversion of the femoral neck $(p<0.01)$, stem sagittal alignment $(p<0.05)$, and body height $(p<0.05)$ were significantly correlated with stem version (Table 2).

Table 2: Radiographic data.

\begin{tabular}{|l|l|l|}
\hline Measurement & Average & Range \\
\hline Anteversion of femoral neck & $26.1 \pm 12.2^{\circ}$ & $-1.0-56.0^{\circ}$ \\
\hline Version of stem & $32.3 \pm 9.0$ & $11.7-55.6^{\circ}$ \\
\hline Sagittal alignment of stem & $1.3 \pm 9.4$ & $-5.5-9.0$ \\
\hline Coronal alignment of stem & $0.06 \pm 2.2$ & $-6.4-5.4$ \\
\hline Increased version of stem & $6.3 \pm 10.9$ & $-10.6-40.5$ \\
\hline
\end{tabular}

Step-wise multiple linear regression analysis showed that native anteversion of the femoral neck $(p<0.01)$, stem sagittal alignment $(p=0.045)$, and body height $(p=0.048)$ were positively correlated with stem aversion (Table 3 ). A $1^{\circ}$ increase in femoral neck anteversion caused a $0.3725^{\circ}$ increase in stem version. A $1^{\circ}$ increase in sagittal alignment of the stem caused a $0.6592^{\circ}$ increase in the version of the J-Taper stem. A $1-\mathrm{cm}$ increase in body height caused a $0.2164^{\circ}$ increase in the version of the $\mathrm{J}$ Taper stem.

The degree of stem version was significantly increased compared with that of native femoral anteversion $(p<0.01)$. The angle of stem version was increased by $6.3^{\circ} \pm 10.9^{\circ}$ (Table 2 ). Spearman's correlation coefficient test showed that anteversion of the femoral neck $(p<0.01)$ and stem sagittal alignment $(p<0.05)$ were significantly correlated with increased version of stem. Step-wise multiple linear regression analysis showed that native anteversion of the femoral neck $(p<0.01)$, stem sagittal alignment $(p=0.045)$, and body height $(p=0.0475)$ were correlated with increased version of stem (Tables 3 and 4 ).

Table 3: Multivariate analysis for the version of the J-Taper stem.

\begin{tabular}{|l|l|l|}
\hline Parameter & p-value & $\begin{array}{l}\text { Partial regression } \\
\text { coefficient }\end{array}$ \\
\hline Body height & 0.0475 & 0.2164 \\
\hline Anteversion of femoral neck & 0.0000 & 0.3725 \\
\hline Sagittal alignment of stem & 0.0450 & 0.6592 \\
\hline
\end{tabular}

Table 4: Multivariate analysis for the increased version of the JTaper stem.

\begin{tabular}{|l|l|l|}
\hline Parameter & $\begin{array}{l}\mathbf{p}- \\
\text { value }\end{array}$ & $\begin{array}{l}\text { Partial regression } \\
\text { coefficient }\end{array}$ \\
\hline Body height & $\begin{array}{l}0.047 \\
5\end{array}$ & 0.2164 \\
\hline Anteversion of femoral neck & $\begin{array}{l}0.000 \\
0\end{array}$ & -0.6275 \\
\hline Sagittal alignment of stem & $\begin{array}{l}0.045 \\
0\end{array}$ & 0.6592 \\
\hline
\end{tabular}

\section{Discussion}

Because femoral anteversion has a wide range of variability [13-15], the acetabular component and stem should be placed to optimize the combined anteversion $[3,4]$. It has been reported that version of cementless stems, including the short tapered stem, is increased compared with femoral anteversion $[12,13,16-18]$. Our findings also showed that the version of the $\mathrm{J}$-Taper stem is increased. To the best of our knowledge, this is the first report to evaluate the anteversion of J-Taper. We showed that the version of J-Taper is affected by anteversion of the femoral neck. Additionally, anteversion of the femoral neck was negatively associated with increased stem version compared with anteversion of the femoral neck.

Before the operation, we measured anteversion of the femoral neck by CT. When anteversion of the femoral neck was severe, we tried to decrease the stem version compared with anteversion of the femoral neck as much as possible to achieve 
optimum total anteversion. This negative correlation between the increased stem version and greater anteversion of the femoral neck might be caused by liberalization of the J-Taper placement. The version of metaphyseal-fit cementless stems is defined by the canal version near the level of the center of the lesser trochanter [16]. It might be easier to decrease the anteversion with J-Taper than with the metaphyseal-fit cementless stem.

We showed that version of the J-Taper stem was affected by body height. It has been reported that anteversion of the femur decreases with age in both normal hips and those with congenital dislocations [17]. These findings indicated that femoral growth decreases the anteversion of the femoral neck. We hypothesized that the relation between version of the JTaper stem and body height might be caused by the relation between femoral neck anteversion and body height or the length of the femur. Pearson's correlation coefficient test showed that although there are high relation between length of femur and body height $(p<0.01)$, there were no relation between length of femur and femoral neck anteversion. In addition, multiple linear regression analysis showed that there was no statistically significant relation between the length of the femur and version of J-Taper when length of femur was used as variable instead of body height (data not shown). Further examinations are needed to elucidate the relation between version of the J-Taper stem and body height.

We showed that version of the J-Taper stem was affected by the stem's sagittal alignment. It has been reported that the stem's sagittal alignment affects the version of cementless stems. When the stem is placed at the anterior portion of the femoral neck, the anterior wall of the neck interferes with the stem version [16]. The version of the J-Taper stem might also be affected by the anterior wall of the femoral neck, similar to other cementless stems. Although it is unclear whether the sagittal alignment of a cementless stem has an effect on the dislocation rate or long-term results, surgeons should be aware that it alters the stem version vs. the native version of the femoral canal.

There are several limitations in this study. First, we grouped osteoarthritis, osteonecrosis of the femoral head, rapidly destructive coxopathy, and rheumatoid arthritis into a single category of primary diagnoses. Our study had a high proportion of hips with developmental dysplasia. There are usually a higher proportion of hips with primary osteoarthritis than with developmental dysplasia in Europe and the United States $[18,19]$. Because developmental dysplasia of the hip has greater femoral neck anteversion, the mean stem version and the mean increased angle of stem version would be different in these countries. All operations were performed using a posterolateral approach because the anterior approach affects the stem's sagittal alignment [12]. The alignment and version of the J-Taper stem are affected by the approach.

It has been reported that the stem version of the metaphyseal fit stem approximates canal version near the level of the center of the lesser trochanter [16]. The J-Taper stem was developed as thick square shape at the distal portion to achieve rotational stability. In all 59 cases, the distal square portion of the J-Taper stem made contact with the femoral cortex at more than three points (average 5.5) (data not shown). This design concept might affect the version and stem alignment of the J-Taper stem. These findings also suggest that the J-Taper stem achieved primary rotational stability. In addition, J-Taper stem showed no revision and favorable results in all cases.

\section{Conclusion}

The version of the J-Taper stem was significantly correlated with anteversion of the femoral neck, stem sagittal alignment, and body height. The degree of stem version was significantly increased compared with that of native femoral anteversion. Femoral neck anteversion was negatively correlated, and stem sagittal alignment was positively correlated, with an increased angle of stem version. These findings will be helpful to surgeons for preoperative planning and to achieve the appropriate combined anteversion.

\section{Acknowledgement}

We are grateful to Yuji Nakashima for excellent assistance to construct 3D-CT.

\section{References}

1. Daines BK, Dennis DA (2012) The importance of acetabular component position in total hip arthroplasty. Orthop Clin North Am 43: e23-34.

2. Callanan MC, Jarrett B, Bragdon CR, Zurakowski D, Rubash HE, et al. (2011) The John Charnley Award: risk factors for cup malpositioning: quality improvement through a joint registry at a tertiary hospital. Clin Orthop Relat Res 469: 319-329.

3. Widmer KH, Zurfluh B (2004) Compliant positioning of total hip components for optimal range of motion. J Orthop Res 22: $815-821$.

4. Yoshimine $F(2006)$ The safe-zones for combined cup and neck anteversions that fulfill the essential range of motion and their optimum combination in total hip replacements. J Biomech 39: 1315-1323.

5. Chen HH, Morrey BF, An KN, Luo ZP (2009) Bone remodeling characteristics of a short-stemmed total hip replacement. J Arthroplasty 24: 945-950.

6. Morrey BF (1989) Short-stemmed uncemented femoral component for primary hip arthroplasty. Clin Orthop Relat Res: 169-175.

7. Khanuja HS, Banerjee S, Jain D, Pivec R, Mont MA (2014) Short bone-conserving stems in cementless hip arthroplasty. J Bone Joint Surg Am 96: 1742-1752.

8. Burt CF, Garvin KL, Otterberg ET, Jardon OM (1998) A femoral component inserted without cement in total hip arthroplasty. A study of the Tri-Lock component with an average ten-year duration of follow-up. J Bone Joint Surg Am 80: 952-960.

9. Hozack W, Gardiner R, Hearn S, Eng K, Rothman R (1994) Taperloc femoral component. A 2-6-year study of the first 100 consecutive cases. J Arthroplasty 9: 489-493. 
10. Patel RM, Smith MC, Woodward CC, Stulberg SD (2012) Stable fixation of short-stem femoral implants in patients 70 years and older. Clin Orthop Relat Res 470: 442-449.

11. Patel RM, Lo WM, Cayo MA, Dolan MM, Stulberg SD (2013) Stable, dependable fixation of short-stem femoral implants at 5 years. Orthopedics 36: e301-307.

12. Abe H, Sakai T, Takao M, Nishii T, Nakamura N, et al. (2015) Difference in Stem Alignment Between the Direct Anterior Approach and the Posterolateral Approach in Total Hip Arthroplasty. J Arthroplasty 30: 1761-1766.

13. Maruyama M, Feinberg JR, Capello WN, D'Antonio JA (2001) The Frank Stinchfield Award: Morphologic features of the acetabulum and femur: anteversion angle and implant positioning. Clin Orthop Relat Res: 52-65.

14. Botser IB, Ozoude GC, Martin DE, Siddiqi AJ, Kuppuswami S, et al. (2012) Femoral anteversion in the hip: comparison of measurement by computed tomography, magnetic resonance imaging, and physical examination. Arthroscopy 28: 619-627.

15. Akiyama M, Nakashima Y, Fujii M, Sato T, Yamamoto T, et al. (2012) Femoral anteversion is correlated with acetabular version and coverage in Asian women with anterior and global deficient subgroups of hip dysplasia: a CT study. Skeletal Radiol 41: 1411-1418.

16. Hirata M, Nakashima $Y$, Itokawa T, Ohishi M, Sato T, et al. (2014) Influencing factors for the increased stem version compared to the native femur in cementless total hip arthroplasty. Int Orthop 38: 1341-1346.

17. Fabry G, MacEwen GD, Shands AR, (1973) Torsion of the femur. A follow-up study in normal and abnormal conditions. J Bone Joint Surg Am 55: 1726-1738.

18. Bargar WL, Jamali AA, Nejad AH (2010) Femoral anteversion in THA and its lack of correlation with native acetabular anteversion. Clin Orthop Relat Res 468: 527-532.

19. Dorr LD, Wan Z, Malik A, Zhu J, Dastane M, et al. (2009) A comparison of surgeon estimation and computed tomographic measurement of femoral component anteversion in cementless total hip arthroplasty. J Bone Joint Surg Am 91: 2598-2604. 